ISSN 1678-3921

Journal homepage: www.embrapa.br/pab

For manuscript submission and journal contents, access: www.scielo.br/pab

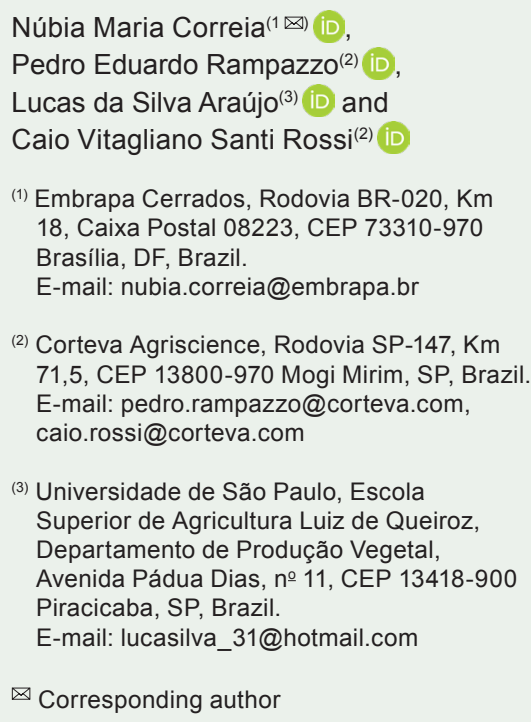

(3) Universidade de São Paulo, Escola Superior de Agricultura Luiz de Queiroz, Departamento de Produção Vegetal, Avenida Pádua Dias, no 11, CEP 13418-900 Piracicaba, SP, Brazil.

E-mail: lucasilva_31@hotmail.com

$\bowtie$ Corresponding author

Received

July 18, 2019

Accepted

June 15, 2020

How to cite

CORREIA, N.M.; RAMPAZZO, P.E.; ARAÚJO,

L. da S.; ROSSI, C.V.S. Sensitivity of Digitaria insularis to herbicides in agricultural areas, in the Brazilian Cerrado biome. Pesquisa Agropecuária Brasileira, v.55, e01570, 2020. DOI: https://doi.org/10.1590/S1678-3921. pab2020.v55.01570.

\section{Sensitivity of Digitaria insularis to herbicides in agricultural areas, in the Brazilian Cerrado biome}

\begin{abstract}
The objective of this work was to evaluate the sensitivity of different populations of Digitaria insularis to the glyphosate, clethodim, and haloxyfop-P-methyl herbicides, in agricultural areas, and to develop infestation maps based on the responses of these populations. One hundred sixty-one populations suspected of being resistant were evaluated and compared to a susceptible population. When plants displayed three to four tillers, the populations were sprayed with glyphosate $\left(1,000 \mathrm{~g} \mathrm{ha}^{-1}\right.$ a.e. $)$, clethodim $(108 \mathrm{~g}$ $\mathrm{ha}^{-1}$ a.i. $+0.5 \%$ mineral oil), and haloxyfop-P-methyl (62.35 $\mathrm{g} \mathrm{ha}^{-1}$ a.i. $+0.5 \%$ mineral oil); plants without herbicide application were used as the control. The plant populations were classified as susceptible, intermediately resistant (with susceptible and resistant plants), or resistant to the tested herbicides. All populations were susceptible to clethodim; $97.5 \%$ were susceptible and $2.5 \%$ were intermediately resistant to haloxyfop-P-methyl; and $9.9 \%$ were susceptible, $21.1 \%$ intermediately resistant, and $68.9 \%$ resistant to glyphosate. Glyphosate-resistant populations are homogeneously distributed throughout the evaluated regions. There are no cases of $D$. insularis multiple resistance in the sampled regions; however, cross-resistance to glyphosate and haloxyfopP-methyl was detected.
\end{abstract}

Index terms: clethodim, glyphosate, haloxyfop-P-methyl, sourgrass, weed.

\section{Sensibilidade de Digitaria insularis a herbicidas em áreas agrícolas no bioma Cerrado brasileiro}

Resumo - O objetivo deste trabalho foi avaliar a sensibilidade de diferentes populações de Digitaria insularis aos herbicidas glifosato, cletodim e haloxifope-P-metílico, em áreas agrícolas, e elaborar mapas de infestação com base na resposta das populações aos herbicidas. Cento e sessenta e uma populações com suspeita de serem resistentes foram avaliadas e comparadas a uma população suscetível. Quando as plantas estavam com três a quatro perfilhos, as populações foram pulverizadas com glifosato (1.000 $\mathrm{g} \mathrm{ha}^{-1} \mathrm{e} . \mathrm{a}$.), cletodim (108 $\mathrm{g} \mathrm{ha}^{-1}$ i.a. $+0,5 \%$ de óleo mineral) e com haloxifope-P-metílico $\left(62,35 \mathrm{~g} \mathrm{ha}^{-1}\right.$ i.a. $+0,5 \%$ de óleo mineral); plantas sem aplicação de herbicidas foram usadas como controle. As populações foram classificadas como sensíveis, resistentes ou intermediárias (com plantas sensíveis e resistentes) aos herbicidas-teste. Todas as populações foram sensíveis ao cletodim; 97,5\% foram sensíveis e 2,5\% intermediárias a haloxifope-P-metílico; e 9,9\% foram sensíveis, $21,1 \%$ intermediárias e $68,9 \%$ resistentes ao glifosato. Populações resistentes ao herbicida glifosato estão dispersas homogeneamente nas regiões avaliadas. Não há casos de resistência múltipla de $D$. insularis nas regiões amostradas; no entanto, detectou-se resistência cruzada a glifosato e a haloxifope-P-metílico.

Termos para indexação: cletodim, glifosato, haloxifope-P-metílico, capimamargoso, plantas daninhas. 


\section{Introduction}

Digitaria insularis (L.) Fedde is a perennial, herbaceous, erect weed species that forms tufts of short rhizomes, and reproduces by seed that are covered by hairs; thus, they are carried by the wind to great distances (Kissmann \& Groth, 1997). Resistant biotypes of $D$. insularis were selected in agricultural areas due to the successive and intense use of the glyphosate herbicide. In Brazil, the resistance of this species to glyphosate was first reported in 2008, in the state of Paraná (Heap, 2020). The first case of $D$. insularis resistance to acetyl-CoA carboxylase (ACCase) inhibitor of herbicides from the aryloxyphenoxypropionate group (fenoxaprop-P-ethyl and haloxyfop-P-methyl) was reported in 2016 (Heap, 2020).

The infestation of resistant biotypes of $D$. insularis to glyphosate has increased in agricultural areas in the Brazilian Cerrado biome (Lucio et al., 2019). This can result in increases of the production costs because of the need to adopt other management strategies, or in the yield reduction of the crop of economic interest due to competition with weeds that are not controlled (Gazziero et al., 2019). According to Adegas et al. (2018), the cost of weed management in soybean crop areas with glyphosate-resistant $D$. insularis may increase from 165 to $290 \%$. Resistant $D$. insularis plants are difficult to control due to the reduced chemical control options available (Carpejani \& Oliveira, 2013; Gazola et al., 2016; Zobiole et al., 2016), which not only requires changes of herbicides, but also of the weed management in agricultural areas, from the medium to long term, considering rotations and mixtures of herbicide, and the use of soil cover crops to mitigate the weed resistance and prevent the selection of biotypes with multiple resistance (Marochi et al., 2018).

Field surveys and tests to evaluate the possible resistance to herbicides are important for weed management practices (Norsworthy et al., 2012). Weed monitoring encompasses activities that confirm resistance in geographical areas defined in a single point in time, or over several years (Soteres \& Peterson, 2015). In addition, the monitoring can prevent the dispersal of resistant plants and address regional issues, raising awareness of producers in each region and improving weed management (Lopez Ovejero et al., 2017).
Technical visits to farms and field observations have shown flaws in the chemical control of $D$. insularis in agricultural areas, in the states of Goiás and Minas Gerais, and in the Federal District, in Brazil, and resulted in complains of producers. The survival of $D$. insularis in the field can be an indication of resistance to glyphosate (Gazola et al., 2016; Zobiole et al., 2016; Lucio et al., 2019).

The objective of this work was to evaluate the sensitivity of different populations of $D$. insularis to the glyphosate, clethodim, and haloxyfop-P-methyl herbicides, in agricultural areas, and to develop infestation maps based on the responses of these populations.

\section{Materials and methods}

One hundred sixty-one D. insularis populations were evaluated and compared to a known susceptible population (standard population). Fifty-six municipalities were sampled - 33 in the state of Goiás, 22 in the state of Minas Gerais, and 1 in the Federal District of Brazil. The samples were concentrated in the south and southwest regions of Goiás, and in Triângulo Mineiro, Alto Paranaíba, and northwest regions of Minas Gerais (Table 1).

Most populations (158) were from agricultural areas with summer soybean crops. Out of these, 66 were maintained with weeds in the off-season (fallow), and the rest with crops in the autumn-winter season in the following number of areas: beans (7), vegetables (4), and wheat (3), irrigated by center pivot; and cotton (2), maize (53), millet (11), and sorghum (12), under rainfed conditions. The other two populations were from agricultural areas with coffee cultivation.

Twenty to forty panicles were collected from the largest number of plants possible in infestation spots, in each crop field (sample unit). The panicles were collected when seeds were at the stage in which they were easily detachable from the panicle. The panicles collected in the sampling units were packed in paper bags and identified with the property name, municipality, state, geographic coordinates (latitude and longitude), altitude, and crop history of the last two crop seasons.

The seed samples were sent to the sector of Weed Sciences of Embrapa Hortaliças, in Brasília, DF, Brazil, where the study was developed. The sensitivity 
Table 1. Municipality, state, geographic coordinates, altitude, and crops of the agriculture areas of each population (Pop.) of Digitaria insularis.

\begin{tabular}{|c|c|c|c|c|c|}
\hline Pop. & Municipality & State $^{(1)}$ & Geographic coordinates & Altitude (m) & Crop (summer - off-season) \\
\hline 1 & Lagoa Grande & MG & $17^{\circ} 76^{\prime} 05^{\prime \prime} \mathrm{S}, 46^{\circ} 53^{\prime} 73^{\prime \prime} \mathrm{W}$ & 557 & Soybean-fallow \\
\hline 2 & Monte Alegre & MG & $19^{\circ} 00^{\prime} 85^{\prime \prime} \mathrm{S}, 48^{\circ} 58^{\prime} 77^{\prime \prime} \mathrm{W}$ & 702 & Soybean-fallow \\
\hline 3 & Uberlândia & MG & $18^{\circ} 51^{\prime} 42^{\prime \prime} \mathrm{S}, 48^{\circ} 26^{\prime} 40^{\prime \prime} \mathrm{W}$ & 828 & Soybean-fallow \\
\hline 4 & Uberlândia & MG & $18^{\circ} 50^{\prime} 89^{\prime \prime} \mathrm{S}, 48^{\circ} 25^{\prime} 65^{\prime \prime} \mathrm{W}$ & 833 & Soybean-fallow \\
\hline 5 & Padre Bernardo & GO & $15^{\circ} 19^{\prime} 00^{\prime \prime} \mathrm{S}, 48^{\circ} 22^{\prime} 30^{\prime \prime} \mathrm{W}$ & 700 & Soybean-fallow \\
\hline 6 & Tupaciguara & MG & $18^{\circ} 35^{\prime} 04^{\prime \prime} \mathrm{S}, 48^{\circ} 51^{\prime} 39^{\prime \prime} \mathrm{W}$ & 877 & Soybean-fallow \\
\hline 7 & Tupaciguara & MG & $18^{\circ} 35^{\prime} 66^{\prime \prime} \mathrm{S}, 48^{\circ} 53^{\prime} 01^{\prime \prime} \mathrm{W}$ & 820 & Soybean-fallow \\
\hline 8 & Buritis & MG & $15^{\circ} 43^{\prime} 77^{\prime \prime} \mathrm{S}, 46^{\circ} 24^{\prime} 98^{\prime \prime} \mathrm{W}$ & 919 & Soybean-fallow \\
\hline 9 & Buritis & MG & $15^{\circ} 43^{\prime} 26^{\prime \prime} \mathrm{S}, 46^{\circ} 25^{\prime} 11^{\prime \prime} \mathrm{W}$ & 920 & Soybean-corn \\
\hline 10 & Buritis & MG & $15^{\circ} 29^{\prime} 79^{\prime \prime} \mathrm{S}, 46^{\circ} 29^{\prime} 79^{\prime \prime} \mathrm{W}$ & 922 & Soybean-corn \\
\hline 11 & Buritis & MG & $15^{\circ} 48^{\prime} 03^{\prime \prime} \mathrm{S}, 46^{\circ} 25^{\prime} 51^{\prime \prime} \mathrm{W}$ & 914 & Soybean-corn \\
\hline 12 & Buritis & MG & $15^{\circ} 42^{\prime} 59^{\prime \prime} \mathrm{S}, 46^{\circ} 21^{\prime} 32^{\prime \prime} \mathrm{W}$ & 907 & Soybean-corn \\
\hline 13 & Chapada Gaúcha & MG & $15^{\circ} 19^{\prime} 05^{\prime \prime} \mathrm{S}, 45^{\circ} 35^{\prime} 47^{\prime \prime} \mathrm{W}$ & 835 & Soybean-fallow \\
\hline 14 & Água Fria de Goiás & GO & $14^{\circ} 48^{\prime} 94^{\prime \prime} \mathrm{S}, 47^{\circ} 39^{\prime} 92^{\prime \prime} \mathrm{W}$ & 1198 & Soybean-fallow \\
\hline 15 & São João da Aliança & GO & $14^{\circ} 42^{\prime} 60^{\prime \prime} \mathrm{S}, 47^{\circ} 35^{\prime} 50^{\prime \prime} \mathrm{W}$ & 1199 & Soybean-fallow \\
\hline 16 & Padre Bernardo & GO & $15^{\circ} 24^{\prime} 51^{\prime \prime} \mathrm{S}, 48^{\circ} 26^{\prime} 34^{\prime \prime} \mathrm{W}$ & 668 & Soybean-fallow \\
\hline 17 & Padre Bernardo & GO & $15^{\circ} 21^{\prime} 45^{\prime \prime} \mathrm{S}, 48^{\circ} 26^{\prime} 33^{\prime \prime} \mathrm{W}$ & 755 & Soybean-fallow \\
\hline 18 & Padre Bernardo & GO & $15^{\circ} 20^{\prime} 00^{\prime \prime} \mathrm{S}, 48^{\circ} 25^{\prime} 13^{\prime \prime} \mathrm{W}$ & 750 & Soybean-fallow \\
\hline 19 & Unaí & MG & $16^{\circ} 02^{\prime} 57^{\prime \prime} \mathrm{S}, 46^{\circ} 30^{\prime} 11^{\prime \prime} \mathrm{W}$ & 940 & Soybean-fallow \\
\hline 20 & Buritis & MG & $15^{\circ} 17^{\prime} 08^{\prime \prime} \mathrm{S}, 46^{\circ} 42^{\prime} 54^{\prime \prime} \mathrm{W}$ & 1008 & Soybean-bean \\
\hline 21 & Buritis & MG & $15^{\circ} 15^{\prime} 59^{\prime \prime} \mathrm{S}, 46^{\circ} 42^{\prime} 20^{\prime \prime} \mathrm{W}$ & 998 & Soybean-fallow \\
\hline 22 & Buritis & MG & $15^{\circ} 49^{\prime} 56^{\prime \prime} \mathrm{S}, 46^{\circ} 23^{\prime} 50^{\prime \prime} \mathrm{W}$ & 950 & Soybean-corn \\
\hline 23 & Buritis & MG & $15^{\circ} 50^{\prime} 30^{\prime \prime} \mathrm{S}, 46^{\circ} 25^{\prime} 21^{\prime \prime} \mathrm{W}$ & 950 & Soybean-corn \\
\hline 24 & Cabeceiras & GO & $15^{\circ} 42^{\prime} 17^{\prime \prime} \mathrm{S}, 47^{\circ} 03^{\prime} 27^{\prime \prime} \mathrm{W}$ & 910 & Soybean-corn \\
\hline 25 & Cabeceiras & GO & $15^{\circ} 47^{\prime} 53^{\prime \prime} \mathrm{S}, 47^{\circ} 05^{\prime} 32^{\prime \prime} \mathrm{W}$ & 920 & Soybean-corn \\
\hline 26 & Planaltina & $\mathrm{DF}$ & $15^{\circ} 47^{\prime} 44^{\prime \prime} \mathrm{S}, 47^{\circ} 24^{\prime} 03^{\prime \prime} \mathrm{W}$ & 910 & Soybean-sorghum \\
\hline 27 & Planaltina & $\mathrm{DF}$ & $15^{\circ} 46^{\prime} 40^{\prime \prime} \mathrm{S}, 47^{\circ} 25^{\prime} 41^{\prime \prime} \mathrm{W}$ & 1000 & Soybean-corn \\
\hline 28 & Catalão & GO & $17^{\circ} 92^{\prime} 67^{\prime \prime} \mathrm{S}, 47^{\circ} 48^{\prime} 87^{\prime \prime} \mathrm{W}$ & 840 & Soybean-corn \\
\hline 29 & Catalão & GO & $17^{\circ} 75^{\prime} 66^{\prime \prime} \mathrm{S}, 47^{\circ} 57^{\prime} 20^{\prime \prime} \mathrm{W}$ & 840 & Soybean-corn \\
\hline 30 & Campo Alegre de Goiás & GO & $17^{\circ} 47^{\prime} 30^{\prime \prime} \mathrm{S}, 47^{\circ} 79^{\prime} 83^{\prime \prime} \mathrm{W}$ & 850 & Soybean-corn \\
\hline 31 & Campo Alegre de Goiás & GO & $17^{\circ} 44^{\prime} 31^{\prime \prime} \mathrm{S}, 47^{\circ} 99^{\prime} 22^{\prime \prime} \mathrm{W}$ & 850 & Soybean-corn \\
\hline 32 & Catalão & GO & $17^{\circ} 95^{\prime} 35^{\prime \prime} \mathrm{S}, 47^{\circ} 40^{\prime} 66^{\prime \prime} \mathrm{W}$ & 850 & Soybean-corn \\
\hline 33 & Catalão & GO & $18^{\circ} 17^{\prime} 44^{\prime \prime} \mathrm{S}, 47^{\circ} 94^{\prime} 15^{\prime \prime} \mathrm{W}$ & 840 & Soybean-corn \\
\hline 34 & Campo Alegre de Goiás & GO & $17^{\circ} 28^{\prime} 43^{\prime \prime} \mathrm{S}, 47^{\circ} 83^{\prime} 29^{\prime \prime} \mathrm{W}$ & 840 & Soybean-corn \\
\hline 35 & Planaltina & GO & $15^{\circ} 13^{\prime} 08^{\prime \prime} \mathrm{S}, 47^{\circ} 35^{\prime} 12^{\prime \prime} \mathrm{W}$ & 1250 & Soybean-fallow \\
\hline 36 & Planaltina & GO & $15^{\circ} 28^{\prime} 49^{\prime \prime} \mathrm{S}, 47^{\circ} 33^{\prime} 12^{\prime \prime} \mathrm{W}$ & 960 & Soybean-fallow \\
\hline 37 & Planaltina & GO & $15^{\circ} 24^{\prime} 07^{\prime \prime} \mathrm{S}, 47^{\circ} 32^{\prime} 09^{\prime \prime} \mathrm{W}$ & 1150 & Soybean-fallow \\
\hline 38 & Formosa & GO & $15^{\circ} 18^{\prime} 20^{\prime \prime} \mathrm{S}, 47^{\circ} 29^{\prime} 45^{\prime \prime} \mathrm{W}$ & 1090 & Soybean-fallow \\
\hline 39 & Planaltina & GO & $15^{\circ} 13^{\prime} 07^{\prime \prime} \mathrm{S}, 47^{\circ} 31^{\prime} 56^{\prime \prime} \mathrm{W}$ & 1190 & Soybean-bean \\
\hline 40 & Planaltina & GO & $15^{\circ} 17^{\prime} 57^{\prime \prime} \mathrm{S}, 47^{\circ} 31^{\prime} 59^{\prime \prime} \mathrm{W}$ & 1210 & Soybean-bean \\
\hline 41 & Montividiu & GO & $17^{\circ} 00^{\prime} 21^{\prime \prime} \mathrm{S}, 51^{\circ} 08^{\prime} 01^{\prime \prime} \mathrm{W}$ & 830 & Soybean-corn \\
\hline 42 & Rio Verde & GO & $17^{\circ} 38^{\prime} 15^{\prime \prime}$ S, $51^{\circ} 08^{\prime} 20^{\prime \prime} \mathrm{W}$ & 880 & Soybean-corn \\
\hline 43 & Rio Verde & GO & $17^{\circ} 48^{\prime} 54^{\prime \prime} \mathrm{S}, 50^{\circ} 53^{\prime} 25^{\prime \prime} \mathrm{W}$ & 730 & Soybean-corn \\
\hline
\end{tabular}

Continuation... 
Table 1. Continuation...

\begin{tabular}{|c|c|c|c|c|c|}
\hline Pop. & Municipality & State $^{(1)}$ & Geographic coordinates & Altitude (m) & Crop (summer - off-season) \\
\hline 44 & Jataí & GO & $18^{\circ} 00^{\prime} 38^{\prime \prime} \mathrm{S}, 52^{\circ} 07^{\prime} 39^{\prime \prime} \mathrm{W}$ & 839 & Soybean-corn \\
\hline 45 & Jataí & GO & $18^{\circ} 09^{\prime} 91^{\prime \prime} \mathrm{S}, 51^{\circ} 52^{\prime} 58^{\prime \prime} \mathrm{W}$ & 847 & Soybean-corn \\
\hline 46 & Jataí & GO & $18^{\circ} 10^{\prime} 31^{\prime \prime} \mathrm{S}, 52^{\circ} 02^{\prime} 31^{\prime \prime} \mathrm{W}$ & 783 & Soybean-corn \\
\hline 47 & Jataí & GO & $17^{\circ} 38^{\prime} 62^{\prime \prime} \mathrm{S}, 51^{\circ} 40^{\prime} 79^{\prime \prime} \mathrm{W}$ & 904 & Soybean-corn \\
\hline 48 & Jataí & GO & $17^{\circ} 54^{\prime} 94^{\prime \prime} \mathrm{S}, 51^{\circ} 46^{\prime} 06^{\prime \prime} \mathrm{W}$ & 707 & Soybean-corn \\
\hline 49 & Paraúna & GO & $16^{\circ} 53^{\prime} 55^{\prime \prime} \mathrm{S}, 50^{\circ} 26^{\prime} 48^{\prime \prime} \mathrm{W}$ & 780 & Soybean-millet \\
\hline 50 & Tuverlândia & GO & $17^{\circ} 51^{\prime} 07^{\prime \prime} \mathrm{S}, 50^{\circ} 17^{\prime} 28^{\prime \prime} \mathrm{W}$ & 620 & Soybean-sorghum \\
\hline 51 & Rio Verde & GO & $18^{\circ} 02^{\prime} 46^{\prime \prime} \mathrm{S}, 51^{\circ} 11^{\prime} 02^{\prime \prime} \mathrm{W}$ & 720 & Soybean-corn \\
\hline 52 & Rio Verde & GO & $17^{\circ} 49^{\prime} 14^{\prime \prime} \mathrm{S}, 51^{\circ} 03^{\prime} 28^{\prime \prime} \mathrm{W}$ & 760 & Soybean-sorghum \\
\hline 53 & Rio Verde & GO & $18^{\circ} 08^{\prime} 58^{\prime \prime} \mathrm{S}, 50^{\circ} 52^{\prime} 21^{\prime \prime} \mathrm{W}$ & 805 & Soybean-millet \\
\hline 54 & Indiara & GO & $17^{\circ} 06^{\prime} 22^{\prime \prime} \mathrm{S}, 49^{\circ} 54^{\prime} 03^{\prime \prime} \mathrm{W}$ & 570 & Soybean-corn \\
\hline 55 & Jandaia & GO & $17^{\circ} 04^{\prime} 00^{\prime \prime} \mathrm{S}, 50^{\circ} 60^{\prime} 10^{\prime \prime} \mathrm{W}$ & 637 & Soybean-sorghum \\
\hline 56 & Acreúna & GO & $17^{\circ} 17^{\prime} 46^{\prime \prime} \mathrm{S}, 50^{\circ} 23^{\prime} 11^{\prime \prime} \mathrm{W}$ & 640 & Soybean-corn \\
\hline 57 & Rio Verde & GO & $17^{\circ} 46^{\prime} 27^{\prime \prime}$ S, 50 54 $14^{\prime} 16^{\prime \prime} \mathrm{W}$ & 760 & Soybean-corn \\
\hline 58 & Rio Verde & GO & $17^{\circ} 42^{\prime} 20^{\prime \prime} \mathrm{S}, 50^{\circ} 58^{\prime} 19^{\prime \prime} \mathrm{W}$ & 770 & Soybean-sorghum \\
\hline 59 & Rio Verde & GO & $17^{\circ} 46^{\prime} 18^{\prime \prime} \mathrm{S}, 50^{\circ} 53^{\prime} 49^{\prime \prime} \mathrm{W}$ & 730 & Soybean-corn \\
\hline 60 & Montividiu & GO & $17^{\circ} 47^{\prime} 37^{\prime \prime} \mathrm{S}, 50^{\circ} 43^{\prime} 40^{\prime \prime} \mathrm{W}$ & 700 & Soybean-sorghum \\
\hline 61 & Santa Helena de Goiás & GO & $17^{\circ} 28^{\prime} 14^{\prime \prime} \mathrm{S}, 51^{\circ} 08^{\prime} 53^{\prime \prime} \mathrm{W}$ & 890 & Soybean-corn \\
\hline 62 & Jataí & GO & $18^{\circ} 02^{\prime} 80^{\prime \prime} \mathrm{S}, 52^{\circ} 01^{\prime} 35^{\prime \prime} \mathrm{W}$ & 877 & Soybean-corn \\
\hline 63 & Jataí & GO & $17^{\circ} 53^{\prime} 78^{\prime \prime} \mathrm{S}, 52^{\circ} 01^{\prime} 76^{\prime \prime} \mathrm{W}$ & 842 & Soybean-corn \\
\hline 64 & Jataí & GO & $18^{\circ} 11^{\prime} 03^{\prime \prime} \mathrm{S}, 51^{\circ} 53^{\prime} 05^{\prime \prime} \mathrm{W}$ & 814 & Soybean-corn \\
\hline 65 & Unaí & MG & $16^{\circ} 43^{\prime} 15^{\prime \prime} \mathrm{S}, 48^{\circ} 43^{\prime} 54^{\prime \prime} \mathrm{W}$ & 547 & Soybean-fallow \\
\hline 66 & Cristalina & GO & $16^{\circ} 20^{\prime} 34^{\prime \prime} \mathrm{S}, 47^{\circ} 25^{\prime} 19^{\prime \prime} \mathrm{W}$ & 859 & Soybean-fallow \\
\hline 67 & Paracatu & MG & $16^{\circ} 39^{\prime} 44^{\prime \prime} \mathrm{S}, 47^{\circ} 02^{\prime} 33^{\prime \prime} \mathrm{W}$ & 898 & Soybean-fallow \\
\hline 68 & Unaí & MG & $16^{\circ} 25^{\prime} 09^{\prime \prime} \mathrm{S}, 47^{\circ} 18^{\prime} 89^{\prime \prime} \mathrm{W}$ & 987 & Soybean-bean \\
\hline 69 & Unaí & MG & $16^{\circ} 29^{\prime} 08^{\prime \prime} \mathrm{S}, 47^{\circ} 29^{\prime} 08^{\prime \prime} \mathrm{W}$ & 847 & Soybean-corn \\
\hline 70 & Paracatu & MG & $16^{\circ} 54^{\prime} 48^{\prime \prime} \mathrm{S}, 47^{\circ} 06^{\prime} 31^{\prime \prime} \mathrm{W}$ & 892 & Coffee \\
\hline 71 & Unaí & MG & $16^{\circ} 27^{\prime} 93^{\prime \prime} \mathrm{S}, 47^{\circ} 07^{\prime} 69^{\prime \prime} \mathrm{W}$ & 978 & Soybean-sorghum \\
\hline 72 & Unaí & MG & $16^{\circ} 34^{\prime} 43^{\prime \prime} \mathrm{S}, 47^{\circ} 15^{\prime} 34^{\prime \prime} \mathrm{W}$ & 934 & Soybean-fallow \\
\hline 73 & Cabeceira Grande & MG & $15^{\circ} 59^{\prime} 17^{\prime \prime} \mathrm{S}, 47^{\circ} 02^{\prime} 79^{\prime \prime} \mathrm{W}$ & 949 & Soybean-corn \\
\hline 74 & Unaí & MG & $16^{\circ} 07^{\prime} 89^{\prime \prime} \mathrm{S}, 46^{\circ} 36^{\prime} 01^{\prime \prime} \mathrm{W}$ & 950 & Soybean-corn \\
\hline 75 & Cabeceira Grande & MG & $15^{\circ} 05^{\prime} 08^{\prime \prime} \mathrm{S}, 47^{\circ} 04^{\prime} 87^{\prime \prime} \mathrm{W}$ & 870 & Soybean-fallow \\
\hline 76 & Unaí & MG & $15^{\circ} 57^{\prime} 79^{\prime \prime} \mathrm{S}, 46^{\circ} 39^{\prime} 78^{\prime \prime} \mathrm{W}$ & 955 & Soybean-sorghum \\
\hline 77 & Unaí & MG & $16^{\circ} 24^{\prime} 42^{\prime \prime} \mathrm{S}, 47^{\circ} 13^{\prime} 94^{\prime \prime} \mathrm{W}$ & 981 & Soybean-fallow \\
\hline 78 & Garapuava & MG & $16^{\circ} 08^{\prime} 38^{\prime \prime} \mathrm{S}, 46^{\circ} 37^{\prime} 02^{\prime \prime} \mathrm{W}$ & 962 & Soybean-fallow \\
\hline 79 & Buriti Alegre & GO & $18^{\circ} 07^{\prime} 63^{\prime \prime} \mathrm{S}, 49^{\circ} 15^{\prime} 14^{\prime \prime} \mathrm{W}$ & 625 & Soybean-corn \\
\hline 80 & Bom Jesus de Goiás & GO & $18^{\circ} 11^{\prime} 87^{\prime \prime} \mathrm{S}, 49^{\circ} 47^{\prime} 68^{\prime \prime} \mathrm{W}$ & 673 & Soybean-corn \\
\hline 81 & Porteirão & GO & $18^{\circ} 00^{\prime} 83^{\prime \prime} \mathrm{S}, 50^{\circ} 07^{\prime} 16^{\prime \prime} \mathrm{W}$ & 507 & Soybean-millet \\
\hline 82 & Goiatuba & GO & $18^{\circ} 00^{\prime} 06^{\prime \prime} \mathrm{S}, 49^{\circ} 36^{\prime} 83^{\prime \prime} \mathrm{W}$ & 723 & Soybean-corn \\
\hline 83 & Edeia & GO & $17^{\circ} 22^{\prime} 10^{\prime \prime} \mathrm{S}, 49^{\circ} 54^{\prime} 61^{\prime \prime} \mathrm{W}$ & 690 & Soybean-millet \\
\hline 84 & Itumbiara & GO & $18^{\circ} 12^{\prime} 36^{\prime \prime} \mathrm{S}, 49^{\circ} 16^{\prime} 16^{\prime \prime} \mathrm{W}$ & 562 & Soybean-corn \\
\hline 85 & Goiatuba & GO & $18^{\circ} 04^{\prime} 25^{\prime \prime} \mathrm{S}, 49^{\circ} 17^{\prime} 58^{\prime \prime} \mathrm{W}$ & 653 & Soybean-sorghum \\
\hline 86 & Joviânia & GO & $17^{\circ} 48^{\prime} 12^{\prime \prime} \mathrm{S}, 49^{\circ} 39^{\prime} 41^{\prime \prime} \mathrm{W}$ & 834 & Soybean-corn \\
\hline
\end{tabular}

Continuation...

Pesq. agropec. bras., Brasília, v.55, e01570, 2020

DOI: 10.1590/S1678-3921.pab2020.v55.01570 
Table 1. Continuation...

\begin{tabular}{|c|c|c|c|c|c|}
\hline Pop. & Municipality & State $^{(1)}$ & Geographic coordinates & Altitude (m) & Crop (summer - off-season) \\
\hline 87 & Cristalina & GO & $16^{\circ} 03^{\prime} 32^{\prime \prime} \mathrm{S}, 47^{\circ} 30^{\prime} 10^{\prime \prime} \mathrm{W}$ & 1020 & Soybean-fallow \\
\hline 88 & Cristalina & GO & $16^{\circ} 38^{\prime} 44^{\prime \prime} \mathrm{S}, 47^{\circ} 34^{\prime} 14^{\prime \prime} \mathrm{W}$ & 1000 & Soybean-sorghum \\
\hline 89 & Cristalina & GO & $16^{\circ} 52^{\prime} 30^{\prime \prime} \mathrm{S}, 47^{\circ} 22^{\prime} 40^{\prime \prime} \mathrm{W}$ & 915 & Soybean-corn \\
\hline 90 & Cristalina & GO & $16^{\circ} 19^{\prime} 06^{\prime \prime} \mathrm{S}, 47^{\circ} 57^{\prime} 01^{\prime \prime} \mathrm{W}$ & 906 & Soybean-bean \\
\hline 91 & Cristalina & GO & $16^{\circ} 49^{\prime} 53^{\prime \prime} \mathrm{S}, 47^{\circ} 44^{\prime} 35^{\prime \prime} \mathrm{W}$ & 1070 & Soybean-corn \\
\hline 92 & Patos de Minas & MG & $18^{\circ} 37^{\prime} 54^{\prime \prime} \mathrm{S}, 46^{\circ} 34^{\prime} 59^{\prime \prime} \mathrm{W}$ & 870 & Soybean-fallow \\
\hline 93 & Patos de Minas & MG & $18^{\circ} 36^{\prime} 43^{\prime \prime} \mathrm{S}, 46^{\circ} 32^{\prime} 26^{\prime \prime} \mathrm{W}$ & 830 & Soybean-fallow \\
\hline 94 & Presidente Olegário & MG & $18^{\circ} 05^{\prime} 44^{\prime \prime} \mathrm{S}, 46^{\circ} 27^{\prime} 52^{\prime \prime} \mathrm{W}$ & 980 & Soybean-fallow \\
\hline 95 & Patrocínio & MG & $18^{\circ} 56^{\prime} 36^{\prime \prime} \mathrm{S}, 47^{\circ} 02^{\prime} 51^{\prime \prime} \mathrm{W}$ & 880 & Soybean-corn \\
\hline 96 & Pedrinópolis & MG & $19^{\circ} 11^{\prime} 46^{\prime \prime} \mathrm{S}, 47^{\circ} 34^{\prime} 21^{\prime \prime} \mathrm{W}$ & 974 & Soybean-wheat \\
\hline 97 & Pedrinópolis & MG & $19^{\circ} 11^{\prime} 10^{\prime \prime} \mathrm{S}, 47^{\circ} 31^{\prime} 10^{\prime \prime} \mathrm{W}$ & 997 & Soybean-fallow \\
\hline 98 & Santa Juliana & MG & $19^{\circ} 30^{\prime} 20^{\prime \prime} \mathrm{S}, 47^{\circ} 25^{\prime} 04^{\prime \prime} \mathrm{W}$ & 976 & Soybean-wheat \\
\hline 99 & Unaí & MG & $16^{\circ} 11^{\prime} 51^{\prime \prime} \mathrm{S}, 46^{\circ} 34^{\prime} 35^{\prime \prime} \mathrm{W}$ & 940 & Soybean-corn \\
\hline 100 & Unaí & MG & $16^{\circ} 10^{\prime} 13^{\prime \prime} \mathrm{S}, 46^{\circ} 37^{\prime} 20^{\prime \prime} \mathrm{W}$ & 950 & Soybean-corn \\
\hline 101 & Unaí & MG & $16^{\circ} 16^{\prime} 36^{\prime \prime} \mathrm{S}, 46^{\circ} 25^{\prime} 20^{\prime \prime} \mathrm{W}$ & 910 & Soybean-corn \\
\hline 102 & Unaí & MG & $16^{\circ} 18^{\prime} 32^{\prime \prime} \mathrm{S}, 46^{\circ} 28^{\prime} 34^{\prime \prime} \mathrm{W}$ & 930 & Soybean-millet \\
\hline 103 & Unaí & MG & $16^{\circ} 23^{\prime} 52^{\prime \prime} \mathrm{S}, 47^{\circ} 14^{\prime} 07^{\prime \prime} \mathrm{W}$ & 970 & Soybean-millet \\
\hline 104 & Conquista & MG & $19^{\circ} 45^{\prime} 36^{\prime \prime} \mathrm{S}, 47^{\circ} 37^{\prime} 01^{\prime \prime} \mathrm{W}$ & 850 & Soybean-fallow \\
\hline 105 & Uberaba & MG & $19^{\circ} 43^{\prime} 50^{\prime \prime} \mathrm{S}, 47^{\circ} 37^{\prime} 46^{\prime \prime} \mathrm{W}$ & 825 & Soybean-fallow \\
\hline 106 & Frutal & MG & $20^{\circ} 06^{\prime} 09^{\prime \prime} \mathrm{S}, 48^{\circ} 55^{\prime} 23^{\prime \prime} \mathrm{W}$ & 520 & Soybean-corn \\
\hline 107 & Uberlândia & MG & $19^{\circ} 05^{\prime} 07^{\prime \prime} \mathrm{S}, 48^{\circ} 12^{\prime} 48^{\prime \prime} \mathrm{W}$ & 930 & Soybean-fallow \\
\hline 108 & Uberlândia & MG & $19^{\circ} 12^{\prime} 44^{\prime \prime} \mathrm{S}, 47^{\circ} 57^{\prime} 00^{\prime \prime} \mathrm{W}$ & 910 & Soybean-fallow \\
\hline 109 & Iraí de Minas & MG & $18^{\circ} 58^{\prime} 02^{\prime \prime} \mathrm{S}, 47^{\circ} 26^{\prime} 45^{\prime \prime} \mathrm{W}$ & 900 & Soybean-fallow \\
\hline 110 & Abadia dos Dourados & MG & $18^{\circ} 27^{\prime} 33^{\prime \prime} \mathrm{S}, 47^{\circ} 22^{\prime} 13^{\prime \prime} \mathrm{W}$ & 850 & Soybean-sorghum \\
\hline 111 & Araguari & MG & $19^{\circ} 33^{\prime} 15^{\prime \prime} \mathrm{S}, 48^{\circ} 20^{\prime} 29^{\prime \prime} \mathrm{W}$ & 900 & Soybean-fallow \\
\hline 112 & Tupaciguara & MG & $18^{\circ} 40^{\prime} 41^{\prime \prime} \mathrm{S}, 48^{\circ} 45^{\prime} 55^{\prime \prime} \mathrm{W}$ & 779 & Soybean-fallow \\
\hline 113 & Tupaciguara & MG & $18^{\circ} 32^{\prime} 05^{\prime \prime} \mathrm{S}, 48^{\circ} 42^{\prime} 25^{\prime \prime} \mathrm{W}$ & 913 & Soybean-fallow \\
\hline 114 & Silvânia & GO & $16^{\circ} 42^{\prime} 20^{\prime \prime} \mathrm{S}, 48^{\circ} 36^{\prime} 41^{\prime \prime} \mathrm{W}$ & 1018 & Soybean-fallow \\
\hline 115 & Palmeiras de Goiás & GO & $16^{\circ} 55^{\prime} 06^{\prime \prime} \mathrm{S}, 49^{\circ} 50^{\prime} 60^{\prime \prime} \mathrm{W}$ & 624 & Soybean-fallow \\
\hline 116 & Palmeiras de Goiás & GO & $16^{\circ} 51^{\prime} 06^{\prime \prime} \mathrm{S}, 49^{\circ} 51^{\prime} 29^{\prime \prime} \mathrm{W}$ & 610 & Soybean-fallow \\
\hline 117 & Morrinhos & GO & $17^{\circ} 43^{\prime} 47^{\prime \prime} \mathrm{S}, 49^{\circ} 01^{\prime} 28^{\prime \prime} \mathrm{W}$ & 861 & Soybean-corn \\
\hline 118 & Orizona & GO & $17^{\circ} 03^{\prime} 32^{\prime \prime} \mathrm{S}, 48^{\circ} 24^{\prime} 10^{\prime \prime} \mathrm{W}$ & 970 & Soybean-fallow \\
\hline 119 & Campinorte & GO & $14^{\circ} 12^{\prime} 09^{\prime \prime} \mathrm{S}, 49^{\circ} 08^{\prime} 50^{\prime \prime} \mathrm{W}$ & 530 & Soybean-corn \\
\hline 120 & Uruaçu & GO & $14^{\circ} 45^{\prime} 46^{\prime \prime} \mathrm{S}, 49^{\circ} 15^{\prime} 50^{\prime \prime} \mathrm{W}$ & 620 & Soybean-corn \\
\hline 121 & Hidrolina & GO & $14^{\circ} 38^{\prime} 58^{\prime \prime} \mathrm{S}, 49^{\circ} 11^{\prime} 09^{\prime \prime} \mathrm{W}$ & 580 & Soybean-sorghum \\
\hline 122 & Hidrolina & GO & $14^{\circ} 47^{\prime} 52^{\prime \prime} \mathrm{S}, 49^{\circ} 17^{\prime} 04^{\prime \prime} \mathrm{W}$ & 550 & Soybean-millet \\
\hline 123 & Uruaçu & GO & $14^{\circ} 27^{\prime} 16^{\prime \prime} \mathrm{S}, 49^{\circ} 09^{\prime} 24^{\prime \prime} \mathrm{W}$ & 770 & Soybean-millet \\
\hline 124 & Padre Bernardo & GO & $15^{\circ} 12^{\prime} 34^{\prime \prime} \mathrm{S}, 48^{\circ} 25^{\prime} 03^{\prime \prime} \mathrm{W}$ & 730 & Soybean-fallow \\
\hline 125 & Padre Bernardo & GO & $15^{\circ} 01^{\prime} 22^{\prime \prime} \mathrm{S}, 48^{\circ} 45^{\prime} 54^{\prime \prime} \mathrm{W}$ & 729 & Soybean-millet \\
\hline 126 & Padre Bernardo & GO & $15^{\circ} 24^{\prime} 66^{\prime \prime} \mathrm{S}, 48^{\circ} 14^{\prime} 93^{\prime \prime} \mathrm{W}$ & 736 & Soybean-fallow \\
\hline 127 & Padre Bernardo & GO & $15^{\circ} 28^{\prime} 04^{\prime \prime} \mathrm{S}, 48^{\circ} 14^{\prime} 65^{\prime \prime} \mathrm{W}$ & 751 & Soybean-fallow \\
\hline 128 & Padre Bernardo & GO & $15^{\circ} 26^{\prime} 21^{\prime \prime} \mathrm{S}, 48^{\circ} 14^{\prime} 89^{\prime \prime} \mathrm{W}$ & 763 & Soybean-fallow \\
\hline 129 & Buritis & MG & $15^{\circ} 38^{\prime} 10^{\prime \prime} \mathrm{S}, 46^{\circ} 27^{\prime} 58^{\prime \prime} \mathrm{W}$ & 952 & Soybean-bean \\
\hline
\end{tabular}

Continuation... 
Table 1. Continuation...

\begin{tabular}{|c|c|c|c|c|c|}
\hline Pop. & Municipality & State $^{(1)}$ & Geographic coordinates & Altitude $(\mathrm{m})$ & Crop (summer - off-season) \\
\hline 130 & Formoso & MG & $14^{\circ} 49^{\prime} 45^{\prime \prime} \mathrm{S}, 46^{\circ} 29^{\prime} 31^{\prime \prime} \mathrm{W}$ & 987 & Soybean-fallow \\
\hline 131 & Formoso & MG & $14^{\circ} 49^{\prime} 07^{\prime \prime} \mathrm{S}, 46^{\circ} 20^{\prime} 99^{\prime \prime} \mathrm{W}$ & 908 & Soybean-fallow \\
\hline 132 & Formoso & MG & $14^{\circ} 48^{\prime} 97^{\prime \prime} \mathrm{S}, 46^{\circ} 20^{\prime} 67^{\prime \prime} \mathrm{W}$ & 917 & Soybean-fallow \\
\hline 133 & Buritis & MG & $15^{\circ} 24^{\prime} 16^{\prime \prime} \mathrm{S}, 46^{\circ} 34^{\prime} 12^{\prime \prime} \mathrm{W}$ & 940 & Soybean-fallow \\
\hline 134 & Buritis & MG & $15^{\circ} 25^{\prime} 46^{\prime \prime} \mathrm{S}, 46^{\circ} 27^{\prime} 02^{\prime \prime} \mathrm{W}$ & 920 & Soybean-fallow \\
\hline 135 & Padre Bernardo & GO & $15^{\circ} 15^{\prime} 34^{\prime \prime} \mathrm{S}, 48^{\circ} 15^{\prime} 44^{\prime \prime} \mathrm{W}$ & 720 & Soybean-fallow \\
\hline 136 & Padre Bernardo & GO & $15^{\circ} 11^{\prime} 24^{\prime \prime} \mathrm{S}, 48^{\circ} 32^{\prime} 00^{\prime \prime} \mathrm{W}$ & 590 & Soybean-fallow \\
\hline 137 & $\begin{array}{l}\text { Santo Antônio do } \\
\text { Descoberto }\end{array}$ & GO & $16^{\circ} 04^{\prime} 31^{\prime \prime} \mathrm{S}, 48^{\circ} 19^{\prime} 09^{\prime \prime} \mathrm{W}$ & 1067 & Soybean-corn \\
\hline 138 & Cristalina & GO & $16^{\circ} 33^{\prime} 37^{\prime \prime} \mathrm{S}, 47^{\circ} 37^{\prime} 00^{\prime \prime} \mathrm{W}$ & 1011 & Coffee \\
\hline 139 & Cristalina & GO & $16^{\circ} 27^{\prime} 54^{\prime \prime} \mathrm{S}, 47^{\circ} 33^{\prime} 35^{\prime \prime} \mathrm{W}$ & 920 & Soybean-fallow \\
\hline 140 & Cristalina & GO & $16^{\circ} 47^{\prime} 25^{\prime \prime} \mathrm{S}, 47^{\circ} 37^{\prime} 59^{\prime \prime} \mathrm{W}$ & 1190 & Soybean-fallow \\
\hline 141 & Unaí & MG & $16^{\circ} 29^{\prime} 41^{\prime \prime} \mathrm{S}, 47^{\circ} 24^{\prime} 53^{\prime \prime} \mathrm{W}$ & 940 & Soybean-fallow \\
\hline 142 & Cristalina & GO & $16^{\circ} 12^{\prime} 05^{\prime \prime} \mathrm{S}, 47^{\circ} 37^{\prime} 48^{\prime \prime} \mathrm{W}$ & 999 & Soybean-cotton \\
\hline 143 & Cristalina & GO & $16^{\circ} 14^{\prime} 23^{\prime \prime} \mathrm{S}, 47^{\circ} 38^{\prime} 06^{\prime \prime} \mathrm{W}$ & 983 & Soybean-cotton \\
\hline 144 & Cristalina & GO & $16^{\circ} 24^{\prime} 31^{\prime \prime} \mathrm{S}, 47^{\circ} 37^{\prime} 40^{\prime \prime} \mathrm{W}$ & 951 & Soybean-tomato \\
\hline 145 & Cristalina & GO & $16^{\circ} 26^{\prime} 37^{\prime \prime} \mathrm{S}, 47^{\circ} 37^{\prime} 36^{\prime \prime} \mathrm{W}$ & 949 & Soybean-tomato \\
\hline 146 & Cristalina & GO & $16^{\circ} 23^{\prime} 34^{\prime \prime} \mathrm{S}, 47^{\circ} 36^{\prime} 42^{\prime \prime} \mathrm{W}$ & 951 & Soybean-fallow \\
\hline 147 & Cristalina & GO & $16^{\circ} 27^{\prime} 28^{\prime \prime} \mathrm{S}, 47^{\circ} 33^{\prime} 51^{\prime \prime} \mathrm{W}$ & 928 & Soybean-fallow \\
\hline 148 & Cristalina & GO & $16^{\circ} 27^{\prime} 08^{\prime \prime} \mathrm{S}, 47^{\circ} 34^{\prime} 02^{\prime \prime} \mathrm{W}$ & 948 & Soybean-fallow \\
\hline 149 & Cristalina & GO & $16^{\circ} 25^{\prime} 19^{\prime \prime} \mathrm{S}, 47^{\circ} 33^{\prime} 41^{\prime \prime} \mathrm{W}$ & 913 & Soybean-millet \\
\hline 150 & Cristalina & GO & $16^{\circ} 24^{\prime} 42^{\prime \prime} \mathrm{S}, 47^{\circ} 33^{\prime} 21^{\prime \prime} \mathrm{W}$ & 886 & Soybean-tomato \\
\hline 151 & Cristalina & GO & $16^{\circ} 25^{\prime} 59^{\prime \prime} \mathrm{S}, 47^{\circ} 35^{\prime} 39^{\prime \prime} \mathrm{W}$ & 881 & Soybean-sweet corn \\
\hline 152 & Cristalina & GO & $16^{\circ} 17^{\prime} 48^{\prime \prime} \mathrm{S}, 47^{\circ} 37^{\prime} 38^{\prime \prime} \mathrm{W}$ & 975 & Soybean-fallow \\
\hline 153 & Cristalina & GO & $16^{\circ} 03^{\prime} 07^{\prime \prime} \mathrm{S}, 47^{\circ} 24^{\prime} 29^{\prime \prime} \mathrm{W}$ & 927 & Soybean-fallow \\
\hline 154 & Planaltina & $\mathrm{DF}$ & $16^{\circ} 01^{\prime} 02^{\prime \prime} \mathrm{S}, 47^{\circ} 26^{\prime} 11^{\prime \prime} \mathrm{W}$ & 954 & Soybean-fallow \\
\hline 155 & Planaltina & $\mathrm{DF}$ & $15^{\circ} 51^{\prime} 29^{\prime \prime} \mathrm{S}, 47^{\circ} 23^{\prime} 14^{\prime \prime} \mathrm{W}$ & 882 & Soybean-wheat \\
\hline 156 & Planaltina & $\mathrm{DF}$ & $15^{\circ} 48^{\prime} 10^{\prime \prime} \mathrm{S}, 47^{\circ} 38^{\prime} 16^{\prime \prime} \mathrm{W}$ & 1034 & Soybean-fallow \\
\hline 157 & Planaltina & $\mathrm{DF}$ & $15^{\circ} 43^{\prime} 29^{\prime \prime} \mathrm{S}, 47^{\circ} 23^{\prime} 21^{\prime \prime} \mathrm{W}$ & 991 & Soybean-corn \\
\hline 158 & Planaltina & $\mathrm{DF}$ & $15^{\circ} 53^{\prime} 15^{\prime \prime} \mathrm{S}, 47^{\circ} 23^{\prime} 29^{\prime \prime} \mathrm{W}$ & 890 & Soybean-bean \\
\hline 159 & Planaltina & $\mathrm{DF}$ & $15^{\circ} 57^{\prime} 48^{\prime \prime} \mathrm{S}, 47^{\circ} 35^{\prime} 24^{\prime \prime} \mathrm{W}$ & 1020 & Soybean-millet \\
\hline 160 & Planaltina & $\mathrm{DF}$ & $16^{\circ} 00^{\prime} 29^{\prime \prime} \mathrm{S}, 47^{\circ} 29^{\prime} 46^{\prime \prime} \mathrm{W}$ & 998 & Soybean-fallow \\
\hline 161 & Uberlândia & MG & $18^{\circ} 53^{\prime} 46^{\prime \prime} \mathrm{S}, 48^{\circ} 17^{\prime} 28^{\prime \prime} \mathrm{W}$ & 865 & Standard population \\
\hline
\end{tabular}

(1)Brazilian federative units: MG, state of Minas Gerais; GO, state of Goiás; and DF, Distrito Federal.

tests were carried out in a greenhouse $\left(15^{\circ} 56^{\prime} 02.10^{\prime \prime} \mathrm{S}\right.$, $48^{\circ} 08^{\prime} 15.94^{\prime \prime} \mathrm{W}$, at $993 \mathrm{~m}$ altitude), under controlled temperature at $30^{\circ} \mathrm{C}$ (day) and $20^{\circ} \mathrm{C}$ (night), and 12-hour photoperiod, from April 2017 to May 2018, using a completely randomized design, with three replicates.

Sixteen sensitivity tests were carried out, according to the receipt of seed samples collected in the field. A standard population (susceptible population to the herbicides) was maintained and used in each test to grade the weed control for comparison. The tested herbicides were the following ones: glyphosate, at 1,000 $\mathrm{g} \mathrm{ha}^{-1}$ of acid equivalent (a.e.); clethodim, at $108 \mathrm{~g} \mathrm{ha}^{-1}$ of the active ingredient (a.i.); and haloxyfop-P-methyl at $62.35 \mathrm{~g} \mathrm{ha}^{-1}$ a.i. A control treatment with no herbicide application was used for each population. The herbicide rates were established based on the recommendation of 
the products for $D$. insularis (Rodrigues \& Almeida, 2018).

D. insularis seed were sown in expanded polystyrene trays for seedling formation. The seedlings were transplanted to pots at 15 days after sowing, and then thinned to maintain two plants only per pot. The experimental unit consisted of $2.0 \mathrm{dm}^{3}$ plastic pots, with a substrate of soil, sand, and plant compost mixture (3:1:1), fertilized with N, P, and K, at 100, 200, and $150 \mathrm{mg} \mathrm{kg}^{-1}$, respectively. Each pot was placed on a plastic container of large diameter, and without holes to maintain the water regime of the plots. Soil moisture was controlled daily, with water replenishing in the containers when necessary.

The herbicides were applied when the plants had 3 to 4 tillers using a $\mathrm{CO}_{2}$ pressurized backpack sprayer with constant-pressure of $2.8 \mathrm{kgf} \mathrm{cm}^{-2}$. The sprayer was equipped with a bar with two flat jet tips (TTI110015) spaced $0.5 \mathrm{~m}$ apart. The application volume was equivalent to $200 \mathrm{~L} \mathrm{ha}^{-1}$.

Visual evaluations of control were carried out at 15 and 30 days after the herbicide application (DAA), using a 0 to $100 \%$ scale of grades, in which zero represents the absence of visual injuries, and 100 represents plant death (Velini et al., 1995). The grades were attributed to each plant of the plot (pot), and the mean control per replicate (pot) was calculated. The mean control was used for the statistical analysis.

Supposedly resistant populations were compared with the susceptible ones and classified as susceptible ( $>80 \%$ control, without regrowth), intermediately resistant (population with susceptible and resistant plants), or resistant (0 to $79 \%$ of control, with regrowth) to the herbicides. Population was classified as intermediate when one resistant plant was found in a plot, even if the other plants in the plot were susceptible. Intermediate resistance is indicative of the existence of resistant individuals in the evaluated population, but not with all plants showing resistance.

The results were used to develop maps of infestation, with the distribution of cases of resistance by species and herbicide in the evaluated municipalities, using the QGIS 2.18 program (Graser \& Peterson, 2016). The maps of the evaluated states [shape files (SHP); 2017 version] were obtained from the website of the Brazilian Institute of Geography and Statistics (IBGE, 2018).
The grades of control (mean values per plot) were subjected to analysis of variance, in order to assess the response variability of weed populations to the tested herbicides. After that procedure, dispersion graphs were developed and the different susceptibility responses to the herbicides were analyzed by hierarchical clustering through the unweighted pair group method with arithmetic mean (UPGMA), using the Toucher method to separate the groups (Silva, 2016).

\section{Results and discussion}

Considering the classification of populations as susceptible, intermediately resistant, and resistant, all weed populations were susceptible to clethodim; $97.5 \%$ were susceptible (Figure 1) and 2.5\% intermediately resistant to haloxyfop-P-methyl (Figure 2); and 9.9\% were susceptible, $21.1 \%$ intermediately resistant, and $68.9 \%$ resistant to glyphosate (Figure 3 ). The results confirmed the dispersion of glyphosate-resistant D. insularis plants in the Cerrado biome, in Brazil. According to Takano et al. (2018), glyphosateresistant $D$. insularis populations can evolve through independent selections, contributing to an expressive dissemination of resistance in agricultural areas. However, despite the survival of $D$. insularis plants in the field, after glyphosate applications, most farmers continue to use only glyphosate for weed management, since effective management strategies require associations of herbicides (Zobiole et al., 2016), which increases the production costs (Adegas et al., 2018).

Populations with intermediate resistance to haloxyfop-P-methyl were found in the municipalities of Abadia dos Dourados (Minas Gerais state), and Montividiu, Padre Bernardo, and Rio Verde (Goiás state). These results raised attention to some areas that were subjected to applications of haloxyfop-P-methyl, since control problems for these populations have been found. The main strategy adopted in agricultural areas for the management of adult $D$. insularis plants resistant to glyphosate is the use of ACCase-inhibitor herbicides, mainly clethodim and haloxyfop-Pmethyl, which are applied at high rates, often above those recommended by the manufacturers, and with sequential applications (Zobiole et al., 2016). This is certainly a problem, since it increases the selection pressure for multiple resistance to glyphosate and ACCase inhibitors, especially haloxyfop-P-methyl, 


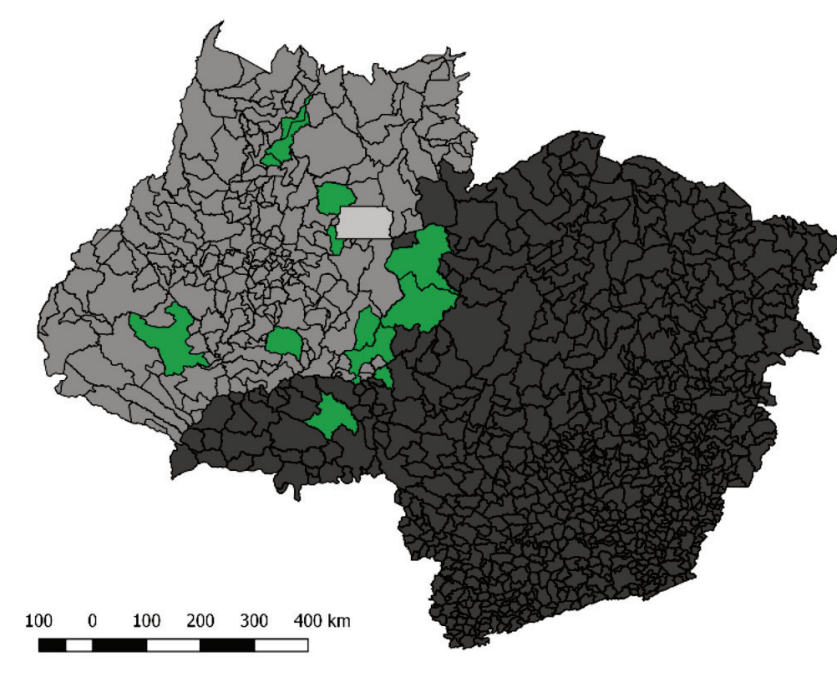

Susceptible

Abadia dos Dourados, MG (1)

Campinorte, GO (1)

Campo Alegre de Goiás, GO (1)

Catalão, GO (1)

Hidrolina, GO (1)

Morrinhos, GO (1)

Padre Bernardo, GO (1)

Paracatu, MG (1)

Rio Verde, GO (2)

Santo Antônio do Descoberto, GO (1)

Uberlândia , MG (1)

Unaí, MG (1)

Uruaçu, GO (2)

Intermediately

Araguari, MG (1)

Catalão, GO (1)

Cristalina, GO (4)

Formoso, MG (2)

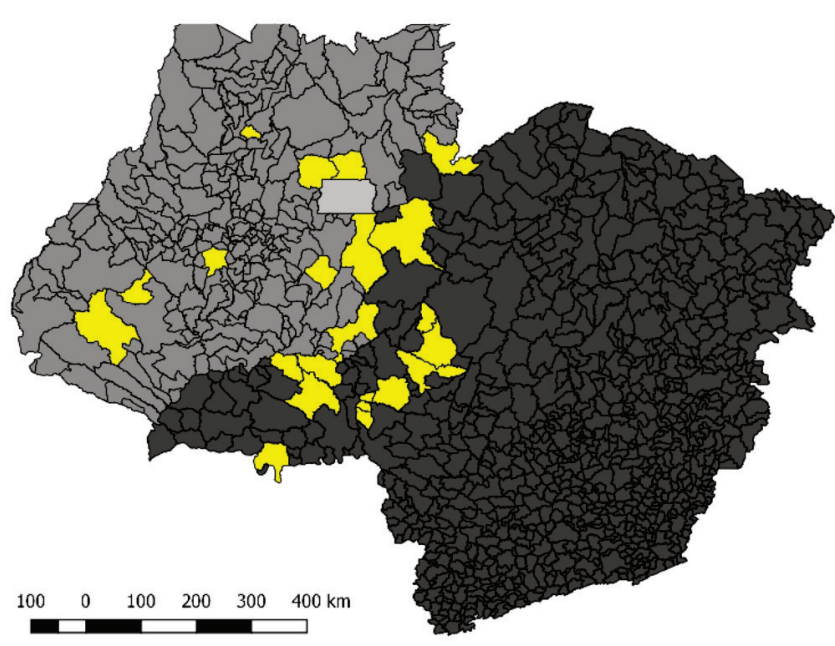

Frutal, MG (1)

Hidrolina, GO (1)

Iraí de Minas, MG (1)

Jataí, GO (2)

Lagoa Grande, MG (1)

Montividiu, GO (1)

Orizona, GO (1)

Padre Bernardo, GO (1)

Palmeiras de Goias, GO (1)

Patos de Minas, MG (2)

Pedrinópolis, MG (2)

Planaltina, GO (1)

Patrocínio, MG (1)

Presidente Olegário, MG (1)

Santa Juliana, MG (1)

Tupaciguara, MG (2)

Uberlândia, MG (4)

Unaí, MG (2)

\section{Resistant}

Acreúna, GO (2)

Água Fria de Goiás, GO (1)

Bom Jesus de Goiás, GO (1)

Buriti Alegre, GO (1)

Buritis, MG (12)

Cabeceira Grande, MG (2)

Cabeceiras, GO (2)

Campo Alegre de Goiás, GO (2)

Catalão, GO (2)

Chapada Gaúcha, MG (1)

Conquista, MG (1)

Cristalina, GO (17)

Edeia, GO (1)

Formosa, GO (1)

Formoso, MG (1)

Goiatuba, GO (2)

Indiara, GO (1)

Itumbiara, GO (1)

Jandaia, GO (1)
Jataí, GO (6)

Joviânia, GO (1)

Monte Alegre de Minas, MG (1)

Montividiu, GO (1)

Padre Bernardo, GO (9)

Palmeiras de Goias, GO (1)

Paracatu, MG (2)

Paraúna, GO (1)

2) Planaltina, DF (9)

Planaltina, GO (4)

Porteirão, GO (1)

Rio Verde, GO (6)

Santa Helena de Goiás, GO (1)

São João da Aliança, GO (1)

Silvânia, GO (1)

Tupaciguara, MG (2)

Tuverlândia, GO (1)

Uberaba, MG (1)

Unaí, MG (10)

Figure 1. Response of Digitaria insularis populations to glyphosate: $9.9 \%$ susceptible (green); $21.1 \%$ intermediately resistant (yellow); and $68.9 \%$ resistant (red). 
as it have occurred for Eleusine indica in Brazil in 2017 (Heap, 2020). Multiple resistance is the ability of plants to survive to applications of herbicides with two or more mechanisms of action (Christoffoleti \& López Ovejero, 2008).

Resistant $D$. insularis to ACCase-inhibitor herbicides in Brazil was caused by the mutation Trp2027Cys in the action site, which does not allow of the connection of several herbicides of the aryloxyphenoxypropionate group to it, such as haloxyfop-P-methyl and fenoxaprop-P-ethyl (Takano et al., 2020). However, all populations were susceptible to clethodim, confirming the importance of this herbicide for the management of $D$. insularis. Moreover, good agricultural practices should be used to avoid the evolution of new cases of resistance and multiple resistance, including the use of soil cover plants, crop rotation, mixture of herbicides, and the association of chemical and mechanical weed control methods (Marochi et al., 2018; Raimondi et al., 2020).

Populations with resistance to glyphosate were found in 38 of the 56 evaluated municipalities, and 10 municipalities showed populations with intermediate resistance, indicating the presence of resistant plants, but at a lower frequency. Thus, more than $85 \%$ of the municipalities displayed already resistant biotypes in at least one of their agricultural areas. These resistant populations were homogeneously distributed in the evaluated regions and not concentrated in a region, or state. Susceptible populations to glyphosate were found in 11 municipalities in the states of Goiás and Minas Gerais. Not all plants that survive to glyphosate applications in the field are resistant to the herbicide. This is explained by the soil moisture conditions and plant size at the time of application. Glyphosate is mainly applied to control adult $D$. insularis plants that show flowers, fruit, and seed (Zobiole et al., 2016; Raimondi et al., 2020), which requires good soil moisture conditions for an adequate absorption, translocation, and action of glyphosate on the plants. Therefore, sometimes the herbicide is not effective due to water stress of plants at the time of application.

According to the analysis of variance, the percentage of control of the $D$. insularis populations was significantly different $(\mathrm{p}<0.01)$, both at 15 and 30 DAA, indicating a variability in their response to

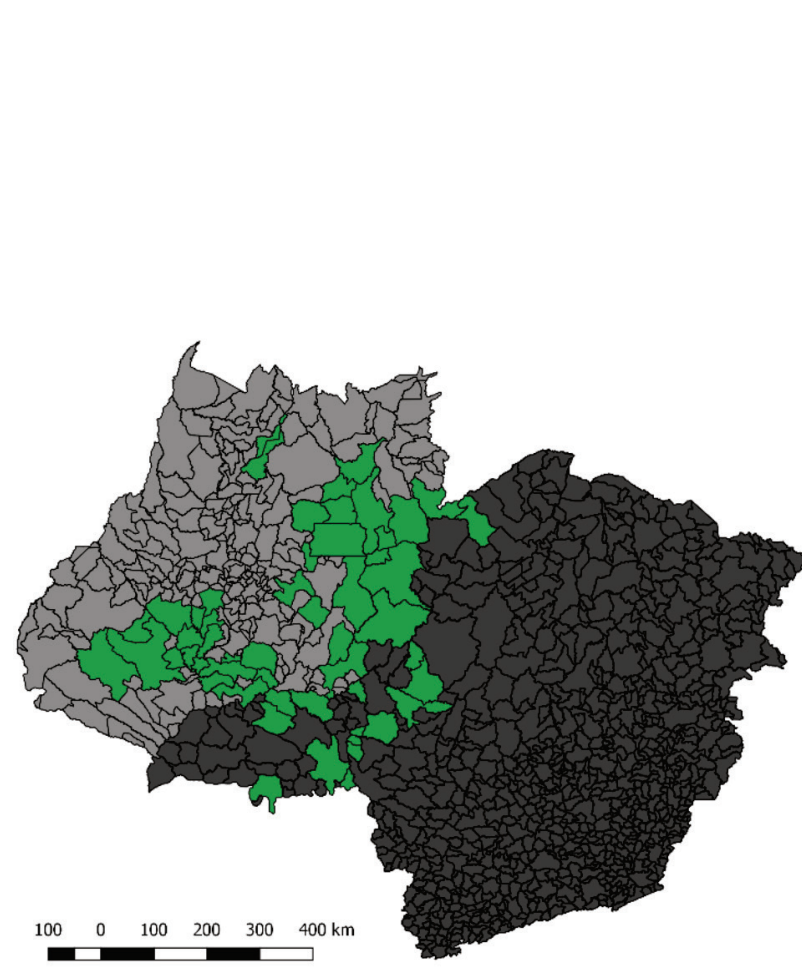

\section{Susceptible}

Abadia dos Dourados, MG (1)

Acreúna, GO (1)

Água Fria de Goiás, GO (1)

Araguari, MG (1)

Bom Jesus de Goiás, GO (1)

Buriti Alegre, GO (1)

Buritis, MG (12)

Cabeceira Grande, MG (2)

Cabeceiras, GO (2)

Campinorte, GO (1)

Catalão, GO (4)

Chapada Gaúcha, MG (1)

Conquista, MG (1)

Cristalina, GO (21)

Edeia, GO (1)

Formosa, GO (1)

Formoso, MG (3)

Frutal, MG (1)

Guarapuava, MG (1)

Goiatuba, GO (2)

Hidrolina, GO (2)

Indiara, GO (1)

Iraí de Minas, MG (1)

Itumbiara, GO (1)

Jandaia, GO (1)

Jataí, GO (8)
Lagoa Grande, MG (1)

Monte Alegre de Minas, MG (1)

Montividiu, GO (2)

Morrinhos, GO (1)

Orizona, GO (1)

Padre Bernardo, GO (11)

Palmeiras de Goiás, GO (2)

Paracatu, MG (2)

Paraúna, GO (1)

Patos de Minas, MG (2)

Pedrinópolis, $\mathrm{MG}(2)$

Planaltina, DF (9)

Planaltina, GO (5)

Porteirão, GO (1)

Patrocínio, MG (1)

Presidente Olegário, MG (1)

Rio Verde, GO (8)

Santa Helena de Goiás, GO (1)

Santa Juliana, MG (1)

Santo Antônio do Descoberto, GO(1)

São João da Aliança, GO (1)

Silvânia, GO (1)

Tupaciguara, MG (4)

Tuverlândia, GO (1)

Uberaba, MG (1)

Unaí, MG (15)

Uruaçu, GO (2)

Figure 2. Response of Digitaria insularis populations to clethodim: 100\% susceptible (green). 
the tested herbicides. Different susceptibility of weed populations to the herbicides was also found through the hierarchical cluster analysis by the Toucher method, which allowed of the formation of 22, 9, and 2 groups of susceptibility to glyphosate, clethodim, and haloxyfop-P-methyl, respectively (Table 2).

The $D$. insularis populations were, in general, not effectively controlled by the glyphosate herbicide, except for 12 populations that showed $\geq 80 \%$ control at $30 \mathrm{DAA}$ (Figure $4 \mathrm{~A}$ ), and fitted to the groups I to $\mathrm{V}$, according to the Toucher's method. The other populations were unsatisfactorily controlled $(<80 \%)$ or not controlled $(0 \%)$, characterizing them as intermediately resistant populations or resistant populations to glyphosate. These populations grouped into 22 groups, most of them (87.6\%) concentrated in the groups VI to XXII, with controls below $80 \%$. The populations in these groups of lower sensitivity showed a high amplitude of response to the herbicide (0 to $79.2 \%$ ).

Eleven populations were the most susceptible ones to the herbicide (Group I), with control grades from $99 \%$ to $100 \%$. These populations were from agricultural areas in the municipalities of Abadia dos Dourados and Unaí, in the state of Minas Gerais; and Campinorte, Hidrolina, Morrinhos, Padre Bernardo, Rio Verde, Santo Antônio do Descoberto, and Uruaçu, in the state of Goiás, Brazil. None of these areas was kept with weeds, with seed production and increase of the soil seed bank between crop seasons. All these areas were cultivated with soybean, in the spring-summer season, combined with other crops in

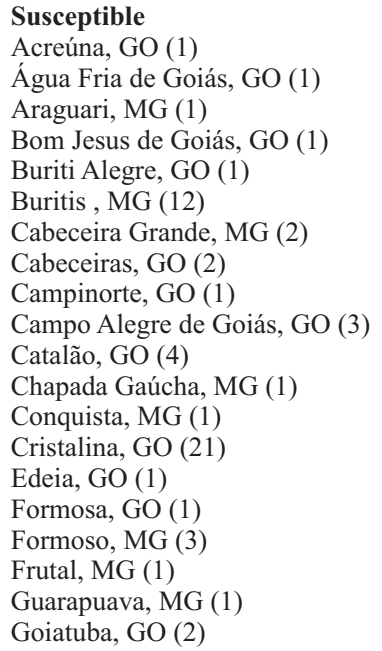

Hidrolina , GO (2) Indiara, GO (1)

IraÍ de Minas, MG (1) Itumbiara, GO (1)

Jandaia, GO (1)

Jataí, GO (8)

Joviânia, GO (1)

Lagoa Grande, MG (1)

Monte Alegre de Minas, MG (1)

Montividiu, GO (2)

Morrinhos, GO (1)

Orizona, GO (1)

Padre Bernardo, GO (10)

Palmeiras de Goiás, GO (2)

Paracatu, MG (2)

Paraúna, GO (1)

Patos de Minas, MG (2)

Pedrinópolis, MG (2)

Planaltina, DF (9)

Planaltina, GO (5)

Porteirão, GO (1)
Patrocínio, MG (1)

Presidente Olegário, MG (1)

Rio Verde, GO (7)

Santa Helena de Goiás, GO (1)

Santa Juliana, MG (1)

Santo Antônio do Descoberto, GO (1)

São João da Aliança, GO (1)

Silvânia, GO (1)

Tupaciguara, MG (4)

Tuverlândia, GO (1)

Uberaba, MG (1)

Uberlândia, MG (5)

Unaí, MG (15)

Uruaçu, GO (2)

Intermediately

Abadia dos Dourados, MG (1)

Montividiu, GO (1)

Padre Bernardo, GO (1)

Rio Verde, GO (1)
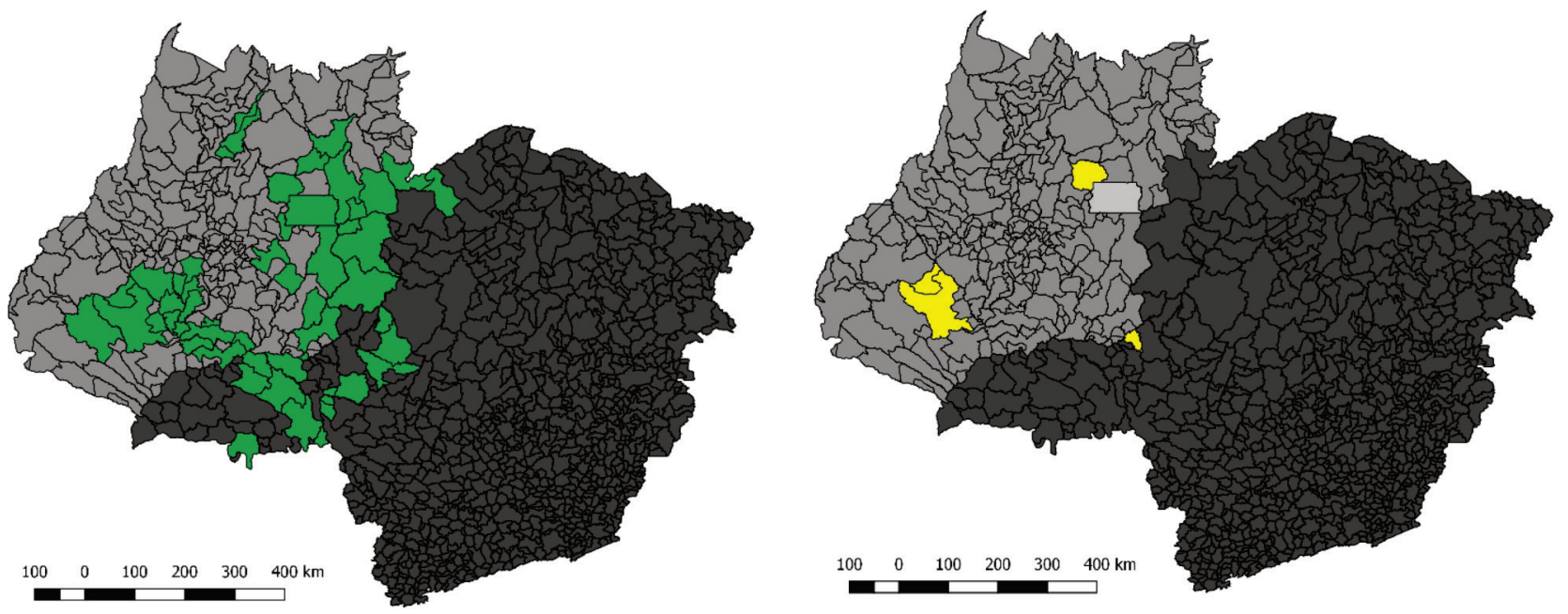

Figure 3. Sensitivity of Digitaria insularis populations to haloxyfop-P-methyl: $97.5 \%$ susceptible (green) and 2.5 intermediately resistant (yellow). 
Table 2. Digitaria insularis grouping of 16 populations as a function of susceptibility to the herbicides glyphosate $(1,000$ $\mathrm{g} \mathrm{a}^{-1}$ a.e.), clethodim (108 $\mathrm{g} \mathrm{ha}^{-1}$ a.i.), and haloxyfop-P-methyl (62.35 $\mathrm{g} \mathrm{ha}^{-1}$ a.i.), using the Toucher's method.

\begin{tabular}{|c|c|c|c|}
\hline Herbicide & Group & Control interval (\%) & Population $^{(1)}$ \\
\hline \multirow{22}{*}{$\sum_{\substack{0 \\
0}}^{\substack{0 \\
0}}$} & I & $99.0-100.0$ & $101,103,110,117,119,120,122,123,137,125,52$ \\
\hline & II & $92.5-95.0$ & $41,69,58$ \\
\hline & III & 89.2 & 32 \\
\hline & IV & 85.0 & 4 \\
\hline & $\mathrm{V}$ & $80.0-82.5$ & $106,113,1$ \\
\hline & VI & $75.0-79.2$ & $2,94,97,99,108,132,95,98,109,116$ \\
\hline & VII & $6.7-71.7$ & $5,145,105,45,89,128,104,90,102,121$ \\
\hline & VIII & $61.7-65.0$ & $88,92,44,31,96$ \\
\hline & XIX & $58.3-60.0$ & 111,140 \\
\hline & $\mathrm{X}$ & $53.3-56.7$ & $86,131,100,93,142,114$ \\
\hline & $\mathrm{XI}$ & 50.0 & $133,134,149$ \\
\hline & XII & $45.0-45.8$ & $107,115,63$ \\
\hline & XIII & $38.3-43.3$ & $7,15,28,39,67,126,127,150,3,156,157,18,87,144$ \\
\hline & XIV & $33.3-36.7$ & $112,155,135,17$ \\
\hline & $\mathrm{XV}$ & 30.0 & 14 \\
\hline & XVI & $21.7-26.7$ & $8,9,36,26,154,59,118$ \\
\hline & XVII & 20.0 & $22,24,56,76,138,153,158$ \\
\hline & XVIII & 16.7 & 10,143 \\
\hline & XIX & 13.3 & $16,66,84,91,130,146$ \\
\hline & $\mathrm{XX}$ & $8.3-10.0$ & $29,37,49,57,60,129,136,139,147,148,43$ \\
\hline & XXI & 6.7 & $12,46,54,77,81,83,33,13,42,50,51,55,65$ \\
\hline & XXII & 0.0 & $\begin{array}{l}6,11,19,20,21,23,25,27,30,34,35,38,40,47,48,53,61,62,64,68,70,71,72,73,74,75, \\
78,79,80,82,85,124,141,151,152,159,160\end{array}$ \\
\hline \multirow{9}{*}{$\begin{array}{l}\text { 吾 } \\
\text { 竞 } \\
\frac{\overrightarrow{0}}{0}\end{array}$} & I & $99.2-100.0$ & $\begin{array}{l}1,4,8,12,14,15,19,22,24,27,28,29,32,34,35,37,38,39,40,42,53,56,63,69,70,73, \\
74,75,80,87,88,93,94,95,96,98,99,100,102,107,111,112,113,115,116,117,118,119, \\
120,122,123,136,137,139,140,141,153,157,61,89,90,91,92,103,106,121,128,129, \\
135,161,3,5,101,110,114,125,132,9,23,31,36,43,46,62,64,67,72,108,126,134,156\end{array}$ \\
\hline & II & $98.8-99.0$ & $17,131,133,109,149$ \\
\hline & III & $97.5-98.5$ & $\begin{array}{l}6,11,18,25,30,41,47,49,51,55,65,68,86,124,10,58,105,130,145,7,16,33,44,50,71, \\
76,77,82,85,97,138,104\end{array}$ \\
\hline & IV & 96.7 & $21,26,54,60,83,84,147,155,48$ \\
\hline & $\mathrm{V}$ & 95.8 & $45,57,59,66$ \\
\hline & VI & $94.2-95.0$ & $13,79,81,158,142,52,78,127$ \\
\hline & VII & $92.5-93.3$ & $2,148,160,20,151,159$ \\
\hline & VIII & 91.7 & $143,146,150$ \\
\hline & IX & $90.0-90.8$ & $144,152,154$ \\
\hline \multirow[t]{2}{*}{ 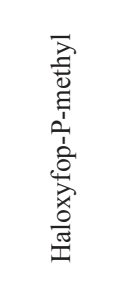 } & I & $89.6-100.0$ & $\begin{array}{l}1,8,75,106,29,74,80,141,5,99,61,108,42,56,69,94,87,114,67,105,22,14,15,62,73, \\
17,70,82,4,109,135,93,64,104,9,122,130,139,140,123,48,11,112,16,43,47,53,23, \\
91,24,50,101,71,97,55,72,83,7,51,49,77,129,10,155,90,85,3,25,98,119,6,124,57, \\
59,68,13,46,44,76,102,113,120,118,54,88,153,103,95,96,84,131,65,63,132,137, \\
18,66,31,60,89,128,26,117,92,121,37,126,12,100,111,19,81,161,20,27,79,134, \\
32,110,127,115,116,138,145,78,86,21,38,45,133,28,107,2,136,58,150,147,149, \\
125,33,158,34,157,30,36,156,159,143,160,152,151,144,40,39,35,146,154,148,142\end{array}$ \\
\hline & II & $16.2-33.3$ & 41,52 \\
\hline
\end{tabular}

\footnotetext{
(1)Populations were organized from the greater control to the lower one, with basis on the group's control interval.
} 

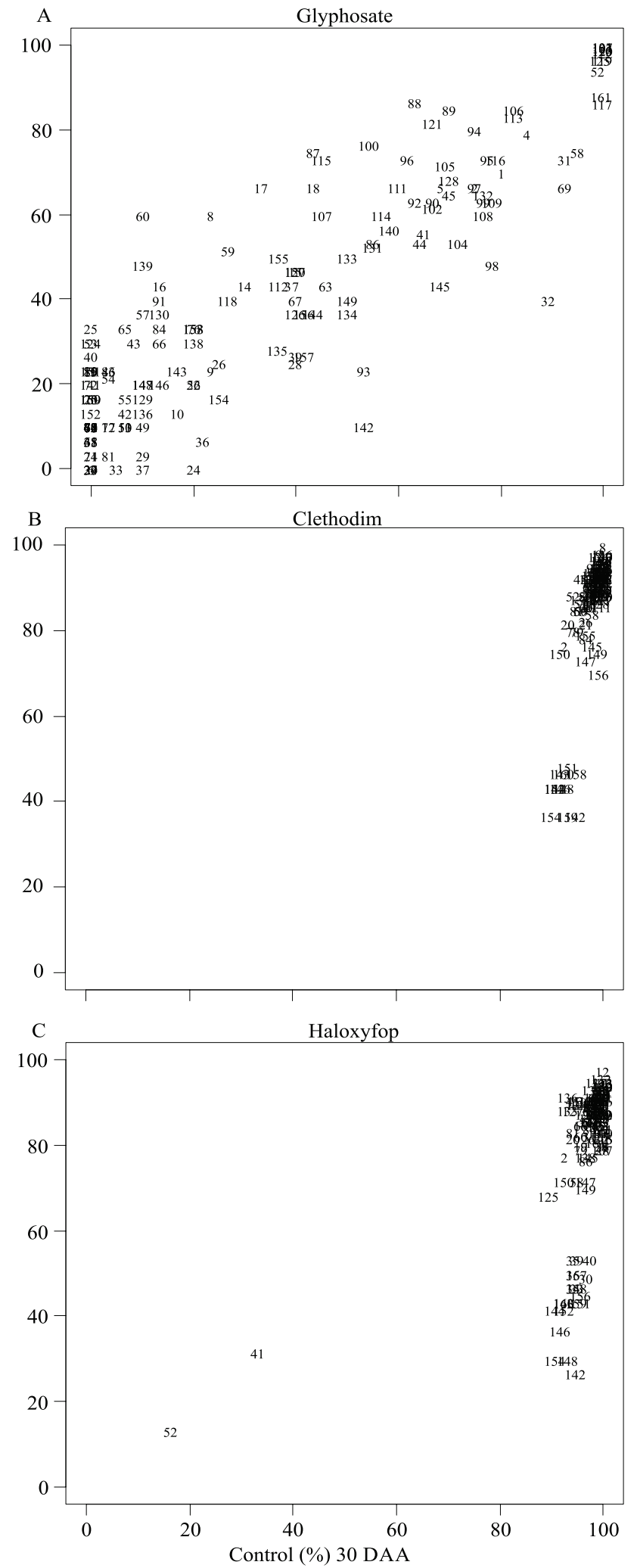

Figure 4. Dispersion of the grade control of Digitaria insularis populations, at 15 and 30 days after application (DAA) of glyphosate (1,000 $\mathrm{g} \mathrm{ha}^{-1}$ a.e.), clethodim (108 $\mathrm{g} \mathrm{ha}^{-1}$ a.i.), and haloxyfop-P-methyl (62.35 $\mathrm{g} \mathrm{ha}^{-1}$ a.i.) herbicides. the autumn-winter season, such as maize, millet, and sorghum, confirming that the best strategy to prevent weed resistance to herbicides is the use of different management strategies, starting by the diversification of crops in the area (Norsworthy et al., 2012; Marochi et al., 2018).

Clethodim displayed an excellent control $(\geq 90 \%)$ of all $D$. insularis populations at 30 DAA. Significant differences were found only at 15 DAA, with 10 populations showing lower-control percentages (Figure 4 B). The Toucher's method showed a highdiscrimination power of the clethodim herbicide, forming nine groups of similar populations. However, the analysis showed a low-dissimilarity degree due to the low variation of control grades between groups. Despite the formation of several groups, the control obtained was satisfactory, and the populations were classified as susceptible.

The susceptibility of the populations to haloxyfopP-methyl was different at 15 DAA for 34 populations, which were more tolerant $(<80 \%$ of control) to this herbicide than the others (Figure $4 \mathrm{C}$ ). However, haloxyfop-P-methyl promoted an excellent control of D. insularis at $30 \mathrm{DAA}$, except for the populations 41 and 52, for which the herbicide was ineffective. This fact was also observed in the analysis by the Toucher's method, which formed two groups: a susceptible group (group I, with 89.6 to $99.7 \%$ control) constituted by $98.8 \%$ of the total evaluated populations; and a tolerant group (group II) with the populations 41 (from Montividiu, with 33.3\% control) and 52 (from Rio Verde, with $16.3 \%$ control).

The map of susceptibility to haloxyfop-P-methyl and the statistical results showed discrepancies. Based on the classification, four populations were intermediately resistant (groups 41, 52, 110, and 125), but only two of them were confirmed by the statistical analysis. This can be explained by the mean of control in the statistics, which was calculated using individual grades of the plants of each replicate. Thus, the control means of the populations 110 and 125, which had only one (110) or two (125) resistant plants, were not significant in comparison to the control means of populations that had four to five resistant plants among the six evaluated ones.

Populations 41, 52, 110, and 125, that had control problems because of haloxyfop-P-methyl applications, showed resistant biotypes; they were susceptible to 
glyphosate and grouped in group I (populations 52, 110, and 125) and group II (population 41), with an excellent control. Therefore, these populations are not related to possible cases of multiple resistance.

\section{Conclusions}

1. The high percentage of resistant Digitaria insularis populations to the herbicide glyphosate are homogeneously distributed throughout the evaluated regions in the Brazilian Cerrado biome.

2 . The sampled regions shows no cases of multiple resistance of $D$. insularis to EPSPs and acetylCoA carboxylase (ACCase) inhibitor herbicides, but displays a cross-resistance to glyphosate and haloxyfop-P-methyl.

\section{Acknowledgments}

To Roni Amaro Bueno, for his assistance in conducting the present work.

\section{References}

ADEGAS, F.S.; GAZZIERO, D.L.P.; VARGAS, L.; KARAM, D.; SILVA, A.F. da; AGOSTINETTO, D. Impacto no bolso. Cultivar: Grandes Culturas, ano18, p.36-38, 2018.

CARPEJANI, M. da S.; OLIVEIRA JR., R.S. de. Manejo químico de capim-amargoso resistente a glyphosate na pré-semeadura da soja. Campo Digital: Revista Ciências Exatas e da Terra e Ciências Agrárias, v.8, p.26-33, 2013.

CHRISTOFFOLETI, P.J.; LÓPEZ OVEJERO, R.F. Resistência das plantas daninhas a herbicidas: definições, bases e situação no Brasil e no mundo. In: CHRISTOFFOLETI, P.J. (Coord.). Aspectos de resistência de plantas daninhas a herbicidas. 3.ed. Piracicaba: Associação Brasileira de Ação a Resistência de Plantas Daninhas aos Herbicidas - HRAC-BR, 2008. p.9-34.

GAZOLA, T.; BELAPART, D.; CASTRO, E.B. de; CIPOLA FILHO, M.L.; DIAS, M.F. Características biológicas de Digitaria insularis que conferem sua resistência à herbicidas e opções de manejo. Científica, v.44, p.557-567, 2016. DOI: https://doi.org/10.15361/1984-5529.2016v44n4p557-567.

GAZZIERO, D.L.P.; ADEGAS, F.S.; SILVA, A.F.; CONCENÇO, G. Estimating yield losses in soybean due to sourgrass interference. Planta Daninha, v.37, e019190835, 2019. DOI: https://doi.org/10.1590/s0100-83582019370100047.

GRASER, A.; PETERSON, G.N. QGIS map design. Chugiak: Locate Press LLC, 2016. 200p.

HEAP, I. The international survey of herbicide resistant weeds. Available at: <http://weedscience.org/Graphs/SOAGraph. aspx> Acessed on: May 252020.
IBGE. Instituto Brasileiro de Geografia e Estatística. Município 2017. Available at: <ftp://geoftp.ibge.gov.br/organizacao_do territorio/malhas_territoriais/malhas_municipais/>. Acessed on: Aug. 102018.

KISSMANN, K.G.; GROTH, D. Plantas infestantes e nocivas. 2.ed. São Paulo: BASF, 1997. 825p. Tomo I.

LOPEZ OVEJERO, R.F.; TAKANO, H.K.; NICOLAI, M.; FERREIRA, A.; MELO, M.S.C.; CAVENAGHI, A.L.; CHRISTOFFOLETI, P.J.; OLIVEIRA JR., R.S. Frequency and dispersal of glyphosate-resistant sourgrass (Digitaria insularis) populations across brazilian agricultural production areas. Weed Science, v.65, p.285-294, 2017. DOI: https://doi.org/10.1017/ wsc. 2016.31.

LUCIO, F.R.; KALSING, A.; ADEGAS, F.S.; ROSSI, C.V.S.; CORREIA, N.M.; GAZZIERO, D.L.P.; SILVA, A.F. da. Dispersal and frequency of glyphosate-resistant and glyphosate-tolerant weeds in soybean-producing edaphoclimatic microregions in Brazil. Weed Technology, v.33, p.217-231, 2019. DOI: https://doi.org/10.1017/wet.2018.97.

MAROCHI, A.; FERREIRA, A.; TAKANO, H.K.; OLIVEIRA JUNIOR, R.S.; LOPEZ OVEJERO, R.F. Managing glyphosateresistant weeds with cover crop associated with herbicide rotation and mixture. Ciência e Agrotecnologia, v.42, p.381-394, 2018. DOI: https://doi.org/10.1590/1413-70542018424017918.

NORSWORTHY,J.K.; WARD, S.M.; SHAW,D.R.;LLEWELLYN, R.S.; NICHOLS, R.L.; WEBSTER, T.M.; BRADLEY, K.W.; FRISVOLD, G.; POWLES, S.B.; BURGOS, N.R.; WITT, W.W., BARRETT, M. Reducing the risks of herbicide resistance: Best management practices and recommendations. Weed Science, v.60, p.31-62, 2012. Special issue. DOI: https://doi.org/10.1614/ WS-D-11-00155.1

RAIMONDI, R.T.; CONSTANTIN, J.; MENDES, R.R.; OLIVEIRA JR., R.S.; RIOS, F.A. Glyphosate-resistant sourgrass management programs associating mowing and herbicides. Planta Daninha, v.38, e020215928, 2020. DOI: https://oi.org/10.1590/ S0100-83582020380100033.

RODRIGUES, B.N.; ALMEIDA, F.S. de. Guia de herbicidas. 7.ed. Londrina: Ed. dos autores, 2018. 764p.

VELINI, E.D.; OSIPE, R.; GAZZIERO, D.L.P. (Coord.). Procedimentos para instalação, avaliação e análise de experimentos com herbicidas. Londrina: SBCPD, 1995. 42p.

SILVA, A.R. da. Métodos de análise multivariada em R. Piracicaba: FEALQ, 2016. 167p.

SOTERES, J.K.; PETERSON, M.A. Industry views of monitoring and mitigation of herbicide resistance. Weed Science, v.63, p.972975, 2015. DOI: https://doi.org/10.1614/WS-D-15-00101.1.

TAKANO, H.K.; OLIVEIRA JR., R.S. de; CONSTANTIN, J.; MANGOLIM, C.A.; MACHADO, M. de F.P.S.; BEVILAQUA, M.R.R. Spread of glyphosate-resistant sourgrass (Digitaria insularis): independent selections or merely propagule dissemination? Weed Biology and Management, v.18, p.50-59, 2018. DOI: https://doi.org/10.1111/wbm.12143. 
TAKANO, H.K.; MELO, M.S.C.; OVEJERO, R.F.L.; WESTRA, P.H.; GAINES, T.A.; DAYAN, F.E. Trp2027Cys mutation evolves in Digitaria insularis with cross-resistance to ACCase inhibitors. Pesticide Biochemistry and Physiology, v.164, p.1-6, 2020. DOI: https://doi.org/10.1016/j.pestbp.2019.12.011.
ZOBIOLE, L.H.S.; KRENCHINSKI, F.H.; ALBRECHT, A.J.P.; PEREIRA, G.; LUCIO, F.R.; ROSSI, C.; RUBIN, R. da S. Controle de capim-amargoso perenizado em pleno florescimento. Revista Brasileira de Herbicidas, v.15, p.157-164, 2016. DOI: http://dx.doi.org/10.7824/rbh.v15i2.474. 